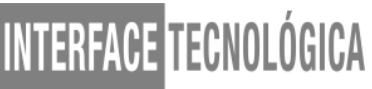

\section{PENSAMENTO COMPUTACIONAL COMO ESTRATÉGIA PARA ELICITAÇÃO REQUISITOS EM DOCUMENTOS}

\author{
COMPUTATIONAL THINKING AS A STRATEGY FOR ELICITATION \\ REQUIREMENTS IN DOCUMENTS
}

\author{
Helena Macedo Reis - helena.macedo@ufpr.br \\ Universidade Federal do Paraná (UFPR) - Jandaia do Sul - Paraná - Brasil \\ Maria Fernanda do Prado Tostes - mfpprado@gmail.com \\ Universidade Estadual do Paraná (UNESPAR) - Paranavaí - Paraná - Brasil \\ Carolina Biserra - carolinabiserra127@outlook.com \\ Universidade Estadual do Paraná (UNESPAR) - Paranavaí - Paraná - Brasil
}

Fernando Tiosso - fernando.tiosso@fatec.sp.gov.br Faculdade de Tecnologia (Fatec) - Taquaritinga - SP - Brasil

Rodrigo Clemente Thom de Souza - thom@ufpr.br Universidade Federal do Paraná (UFPR) - Jandaia do Sul - Paraná - Brasil

DOI: 10.31510/infa.v17i2.891

Data de publicação: 18/12/2020

\section{RESUMO}

A elicitação de requisitos é a primeira etapa da engenharia de requisitos com o objetivo de buscar, levantar, elaborar, negociar, especificar e verificar os serviços e restrições de um sistema. Existem várias técnicas para elicitar requisitos, incluindo análise de documentos. Apesar das vantagens da elicitação por meio da análise documental, existem algumas restrições, como a falta de integração dos dados e a dificuldade de detalhamento das informações. Para minimizar esses problemas, este estudo investigou o uso do Pensamento Computacional para apoiar o levantamento de requisitos. Para verificar a estratégia proposta, os requisitos elicitados foram validados por quatro avaliadores por meio da Revisão de Requisitos e um Protótipo. Os resultados mostraram que os avaliadores consideraram que os requisitos foram eliciados de forma adequada para o desenvolvimento de aplicativos para apoiar os processos pós-cirúrgico perioperatório para enfermagem.

Palavras-chave: Ambientes Interativos de Aprendizagem. Engenharia de Software. Elicitação de Requisitos. 
Requirements elicitation is the first step of requirements engineering with the objective of seeking, surveying, elaborating, negotiating, specifying and verifying the services and restrictions of a system. There are several techniques for eliciting requirements, including document analysis. Despite the advantages of elicitation through document analysis, there are some restrictions, such as the lack of data integration and difficulty in detailing the information. To minimize these problems, this study investigated the use of Computational Thinking to support requirements gathering. To verify the proposed strategy, the elicited requirements were validated by four evaluators through the Requirements Review and a Prototype. The results showed that the evaluators considered that the requirements were adequately elicited for the development of applications to support the perioperative postsurgical processes for nursing.

Keywords: Interactive Learning Environments. Software Engineering. Requirements Analysis.

\section{INTRODUÇÃO}

Sommerville (2011) define requisitos como sendo descrições das necessidades dos clientes, como os serviços o sistema que deve oferecer e as suas restrições. O processo de descobrir, identificar e elaborar requisitos para sistemas computacionais é chamado de elicitação ou levantamento de requisitos (ZOWGHI; COULIN, 2005). De acordo com Pressman e Maxim (2016), entender e realizar elicitação dos requisitos é considerada uma das tarefas mais difíceis de se realizar por um engenheiro de software.

A elicitação de requisitos pode ser feita de diversas formas como por exemplo, entrevistas, cenários, etnografia, entre outros (SOMMERVILLE, 2011). Em sistemas críticos, como os de enfermagem perioperatória pós-cirúrgica ${ }^{1}$, a elicitação de requisitos ocorre mais frequentemente por meio de documentos e protocolos pré-validados para rotinas dos cuidados, elaborados a partir do conhecimento científico atual, respaldados em evidências científicas, por profissionais experientes e especialistas na área.

Além disso, observa-se que somente a técnica de elicitação de requisitos por análise de documentos apresenta baixa necessidade de envolvimento dos usuários e grande quantidade de fontes para consulta de informações, sendo considerada de baixo custo comparada com outras técnicas (DENNIS; WIXOM; ROTH, 2018).

\footnotetext{
${ }^{1}$ A enfermagem perioperatória é uma área de atuação do enfermeiro que abrange a assistência integral ao paciente submetido à cirurgia nos períodos pré-operatório, transoperatório e pós-operatório (SOBECC, 2017). Especialmente, o período pós-cirúrgico abrange as 24 horas após a cirurgia, incluindo o tempo em que o paciente permanece na sala de recuperação pós-anestésica (SRPA) (SOUZA; CARVALHO; PALADINO, 2012).
} 
Apesar das vantagens da elicitação de requisitos por meio da análise de documentos, essa a técnica apresenta algumas desvantagens citada por Dennis, Wixom e Roth (2011): baixo nível de detalhe da informação e baixa integração da informação - o que pode comprometer os requisitos elicitados. Diante destas precupações para análise dos documentos, temos a seguinte questão de pesquisa Q1: É possível aplicar uma estratégia para a análise de documentos para elicitação requisitos que promova maior detalhe e integração da informação? Para buscar responder essa questão de pesquisa, este estudo tem como objetivo propor o Pensamento Computacional como uma estratégia para apoiar as técnicas existentes na elicitação de requisitos em documentos. Para a validação da estratégia, foi desenvolvido um aplicativo para apoiar os procedimentos perioperatórios pós-cirúrgico de enfermagem com requisitos elicitados a partir de documentos pré-validados da área.

Este trabalho encontra-se dividido em seções: na seção 2 são comentados os trabalhos relacionados sobre a elicitação de requisitos por análise de documentos. A seção 3 apresenta o método aplicado para buscar responder a questão de pesquisa. A seção 4 descreve a avaliação da proposta. E finalmente, nas seções 5 e 6 temos as ameaças à validade e a conclusões da pesquisa realizada, respectivamente.

\section{TRABALHOS RELACIONADOS}

A análise de documentos consiste pesquisar de forma qualitativa tópicos de interesse em documentos existentes (BOWEN, 2009). O'Leary (2017) aponta que os documentos como fonte de dados são similares a informações coletadas nas entrevistas, observações e questionários. Elas podem estar em diversos formatos, como fonte de dados imparciais de autoridades (censos, jornais, livros, relatórios e questionários); parciais (materiais promocionais ou de pesquisas produzido por pessoas com interesse nos resultados); comunicação pessoal (e-mails, esboços, fotografias, períodicos, memorandos ou qualquer documento pessoal e subjetivo); multimídia (colunas, artigos de jornais ou revistas, seriados, reportagens) e; documentos históricos (registros, atas e documentos políticos dentro de um período histórico).

Bowen (2009) aponta que a análise de documentos é uma maneira eficiente de coleta de dados, prática e acessível. Geralmente, a elicitação de requisitos por análise de documentos é muito mais econômica comparada com outras técnicas. Além disso, os documentos podem 
ser revisados várias vezes e permanecem inalterados a cada análise do pesquisador. Outra vantagem é que os documentos podem fornecer dados que não podem ser mais observados e mostrar a evolução do sistema.

A análise de documentos envolve atividades de planejar, reunir os documentos, revisar, questionar evidências, refinar e analisar os dados extraídos (O'LEARY, 2017). Na Figura 1 são apresentadas as etapas de verificação para o processo de análise de documento. Após coletar os documentos, verificar sua credibilidade, autenticidade e relevância, a próxima etapa é extrair informações sobre autor, público, tipo do documento, fatos e opiniões. Em seguida, uma exploração de evidências reais no conteúdo do documento é realizada (etapa Questionar), e O'Leary (2017) fornece duas técnicas principais para realizar isso. Uma técnica é a entrevista. Neste caso, o pesquisador trata o documento como o seu entrevistado para fornecer informações relevantes. O pesquisador elabora e "faz" uma série de perguntas, e o pesquisador destaca a resposta no texto. Esta técnica possui algumas desvantagens, como a dificuldade de encontrar informações (detalhamento), pois a mesma informação pode ser escrita de maneiras diferentes. Além disso, é difícil determinar quais requisitos são prioritários e o fluxo das informações (integração).

Figura 1: Etapas para análise de documentos

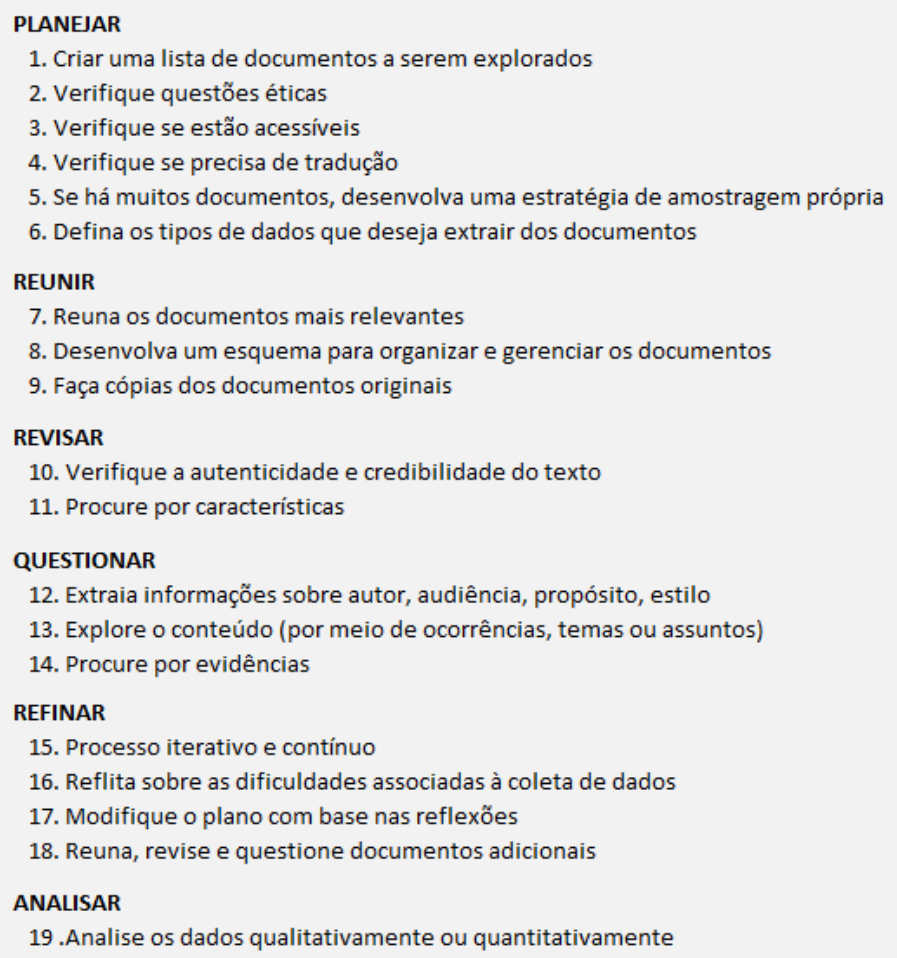

Fonte: O'Leary (2017) 
A outra técnica é quantificar a ocorrências de uma determinada palavra, frase ou conceitos específicos. O pesquisador determina o que vai ser procurado, faz a leitura do documento e registra a quantidade das ocorrências no texto. Para Bowen (2009), esta técnica pode gerar problemas de interpretação, em que algumas informações importantes podem ser omitidas. Entretanto, o autor sugere que a análise de conteúdo pela técnica da quantificação das palavras pode fornecer ao pesquisador informações gerais e identificação de passagens relevantes.

$\mathrm{Na}$ Tabela 1 é apresentada a comparação das técnicas de elicitação de requisitos e como elas influenciam na elaboração ${ }^{2}$ dos requisitos. Observa-se que a técnica "Análise de documento" possui deficiências quanto ao detalhe da informação e integração da informação, apesar de apresentar relativamente baixo custo. Alguns documentos podem apresentar grande quantidade de informações a serem analisadas, sendo difícil encontrar informações essenciais e detalhadas dos requisitos (TSUMAKI; TAMAI, 2006). Além disso, documentos escritos em linguagem natural podem apresentar ambigüidade, sendo que o mesmo dado pode ser escrito de maneira diferente, resultando na dificuldade de integração de requisitos semelhantes. Outros problemas encontrados são: falta de modularização, dificuldade em estruturar requisitos do sistema e dificuldade em definir requisitos prioritários (ZOWGHI; COULIN, 2005).

Tabela 1: Comparação das técnicas de elicitação de requisitos

\begin{tabular}{|l|c|c|c|c|c|}
\cline { 2 - 6 } \multicolumn{1}{c|}{} & Entrevistas & JAD & Questionários & $\begin{array}{c}\text { Análise de } \\
\text { Documentos }\end{array}$ & Observação \\
\hline Detalhe da informação & Alto & Alto & Médio & Baixo & Baixo \\
\hline Fontes da informação & Baixo & Médio & Alto & Alto & Baixo \\
\hline Integração da informação & Baixa & Alta & Baixa & Baixo & Baixo \\
\hline Envolvmento do usuário & Médio & Alta & Baixo & Baixo & Baixo \\
\hline Custo & Médio & Baixo-Médio & Baixo & Baixo & Baixo-Médio \\
\hline
\end{tabular}

Fonte: Dennis, Wixom e Roth (2011)

\footnotetext{
${ }^{2}$ Etapa em que os dados coletados são analisados e refinados
} 


\section{MÉTODO}

\subsection{Q1: É possível aplicar uma estratégia para a análise de documentos para elicitação} requisitos que promova maior detalhe e integração da informação?

Diante dos problemas encontrados na etapa "Questionar", das duas técnicas demonstradas na Seção 2, acredita-se que o Pensamento Computacional (PC) pode auxiliar na elicitação dos requisitos promovendo o detalhamento, integração e fluxo das informações. O Pensamento Computacional consiste em um processo cognitivo onde problemas são resolvidos baseados na lógica, podendo ser aplicado em diferentes artefatos, como processos, procedimentos, problemas, abstrações, objetos, algoritmos ou informações (SELBY; WOOLLARD, 2013; ZOWGHI; COULIN, 2005). Essa abordagem auxilia na resolução de problemas distintos em diferentes contextos, por meio da sistematização, análise de dados e criação de soluções que utilizam uma série de passos ordenados (algoritmos).

Por meio do Pensamento Computacional, é possível resolver problemas, decompondoos em elementos menores e encontrando algoritmos para os resolver. Ela acontece por meio da decomposição (refere-se em dividir um problema em pequenas partes para formular uma solução com mais facilidade), reconhecimento de padrões (é o processo de identificar os aspectos comuns na situação estudada), abstração (refere-se em analisar os elementos relevantes e diferenciando-os dos elementos irrelevantes) e desenho algorítmico (é a solução de um problema por meio de passos e regras para a solução de problemas) (ZIMMERMANN, 2016).

A Tabela 2 apresenta como PC pode auxiliar na coleta de requisitos de forma detalhada e integrada.

Tabela 2: Relação entre PC com detalhes e integração de informações

\begin{tabular}{|l|c|c|}
\cline { 2 - 3 } \multicolumn{1}{c|}{} & Detalhe & Integração \\
\hline Decomposição & $\mathrm{X}$ & \\
\hline Reconhecimento de padrões & & $\mathrm{X}$ \\
\hline Abstração & $\mathrm{X}$ & $\mathrm{X}$ \\
\hline Desenho algorítmico & $\mathrm{X}$ & $\mathrm{X}$ \\
\hline
\end{tabular}

Fonte: Próprio autor 


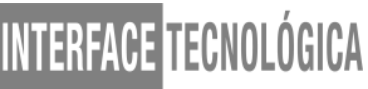

Alguns documentos podem apresentar uma grande quantidade de informação, dificultando o pesquisador de encontrar requisitos importantes. Inicialmente, o pesquisador pode definir o que se deseja encontrar. Pode ser uma questão norteadora ou uma questão semelhante a entrevista. Posteriormente, o pesquisador deve decompor trechos do documento em partes menores, com objetivo de encontrar soluções mais pontuais. Neste momento, o pesquisador pode destacar palavras-chave que resolvam a questão. Após dividir o problema em pequenas partes, o pesquisador deve reconhecer os aspectos comuns dos problemas decompostos e suas soluções. Durante esse processo, o pesquisador também deve identificar os elementos relevantes para aquela solução. Nesta etapa, consequentemente, o pesquisador também estará detalhando aspectos importantes para a solução a ser encontrada. E por fim, o pesquisador deve analisar como as informações encontradas influenciam umas as outras e o fluxo das informações. Nesta etapa, o pesquisador pode apresentar um fluxograma apresentando o desenho algorítmico do fluxo das informações e seus relacionamentos.

A proposta para o uso do PC para análise de documentos altera a etapa Questionar, onde foi adicionado os conceitos de decomposição, reconhecimento de padrões, abstração e desenho algorítmico, para a realização da investigação do documento e a procura por evidências para a elicitação de requisitos.

Com objetivo de validar os itens decompostos, e os requisitos funcionais e não funcionais decompostos ${ }^{3}$, foi desenvolvido um protótipo (Figura 2) para o Sistema Operacional Android com linguagem nativa.

Na Figura 3 são apresentadas as etapas do procedimento para a elicitação de requisitos e desenvolvimento do protótipo para a validação dos requisitos elicitados. Para auxiliar na elicitação de requisitos do aplicativo para resolver o problema geral, foi utilizado o PC em documentos de Assistência de Enfermagem para procedimentos perioperatórios.

\footnotetext{
${ }^{3}$ Todos os requisitos elicitados estão disponíveis em: https://bit.ly/2LjWI8X.
} 
Figura 2: Telas do protótipo.

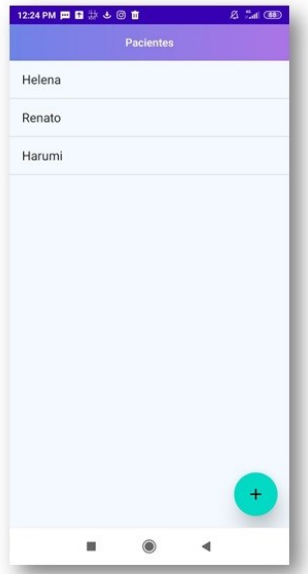

(a)

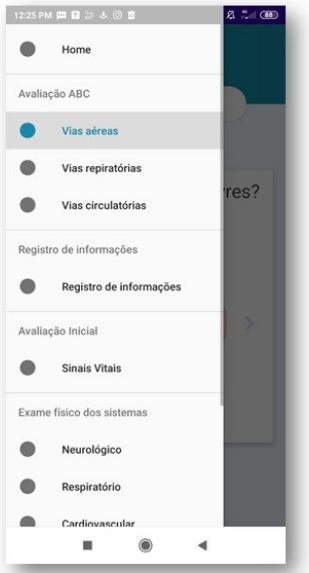

(b)

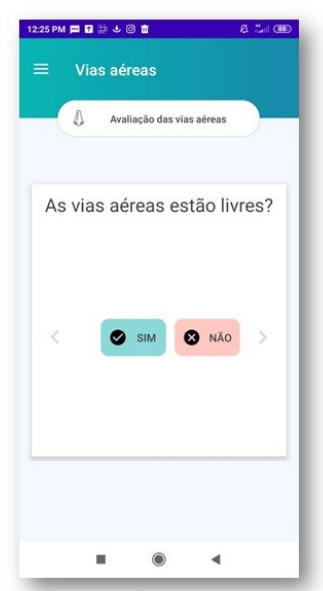

(c)

Fonte: Próprio autor

Os requisitos coletados foram validados, refinados, classificados e organizados por um especialista por meio de entrevista. Nesta etapa, os requisitos poderiam ser consultados novamente nos documentos de saúde. Posteriormente, foi realizado o desenvolvimento de um protótipo para a validação dos requisitos elicitados, passando pelas etapas de definição do objetivo do protótipo, funcionalidades determinadas na elicitação, o desenvolvimento e avaliação. Na avaliação, caso outros requisitos fossem necessários ou ajustados, foi retornado para a etapa de definição das funcionalidades do protótipo.

\section{AVALIAÇÃo}

O processo de validação dos requisitos consiste na verificação dos requisitos com as necessidades do usuário, buscando encontrar problemas na elicitação dos requisitos. Os requisitos são examinados em termos de consistência, omissões e ambiguidade (PRESSMAN; MAXIM, 2016). Sommerville (2011) cita diferentes técnicas para a validação dos requisitos, que podem ser utilizadas individualmente ou em conjunto. Dentre elas, duas técnicas citadas pelo autor e que serão utilizadas para a validação dos requisitos do trabalho proposto é a "Revisão de requisitos" e a "Prototipação". A revisão de requisitos é uma análise sistemática realizada por uma equipe de revisores especialistas que verifica se há inconsistências ou erros. A prototipação consiste na criação de um aplicativo sem funcionalidades inteligentes, como acesso ao banco de dados, para que seja verificado por usuários finais e clientes. 
Figura 3: Processo de elicitação de requisitos, desenvolvimento e avaliação do protótipo

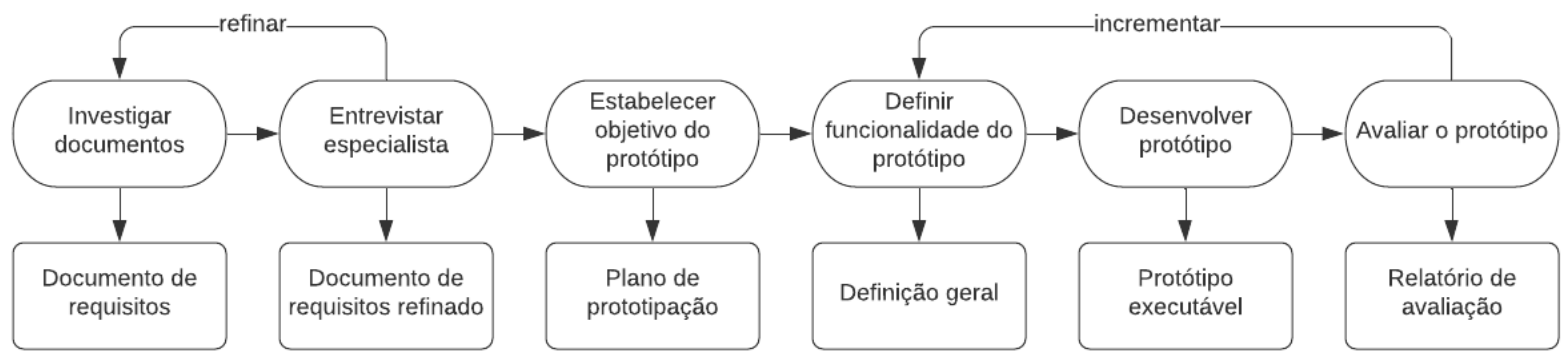

Fonte: Sommerville (2011)

\subsection{Objetivos e Hipóteses}

O propósito dessa avaliação é investigar se o PC é eficiente para a elicitação de requisitos. A partir dessa abordagem, foi formulada as seguintes hipóteses:

- H1: Os avaliadores/juízes apresentaram um alto nível de concordância na Revisão de requisitos dos requisitos elicitados por meio do PC na análise de documentos.

- H2: Os requisitos elicitados apresentados no Protótipo se apresentaram adequados pelos avaliadrores/juízes.

\subsection{Participantes}

Participaram da validação dos requisitos 4 avaliadores graduados em Enfermagem (2 homens e 2 mulheres). Além da graduação, os avaliadores possuem pós-graduação na área, sendo uma doutora, um mestre, um mestrando e uma especialista em Enfermagem (média de 30,5 anos). Dois avaliadores possuem experiência profissional de média de 6,5 e todos são docentes de uma Universidade do Paraná (média de 5 anos).

\subsection{Materiais}

Os materiais utilizados para ao experimento foram: (i) formulário de revisão de requisitos $^{4}$, (ii) formulário para validação do protótipo $^{5}$ e (iii) protótipo. Ambos formulários apresentaram uma introdução sobre o trabalho, quais objetivos, quantidade de questões, tempo médio de participação e um termo de concordância na participação. O formulário de revisão (i) teve como conteúdo baseado proposta de Pressman e Maxim (2016). O formulário cobriu 71 questões, sendo 43 questões abertas e 28 de múltipla escolha, dividido em 7 seções. Cada seção possuiu em 10 questões e um anexo com os requisitos de cada procedimento

\footnotetext{
${ }^{4}$ Disponível em: https://forms.gle/PYZ9j1Rp3xmrgKTX6

${ }^{5}$ Diposnível em: https://forms.gle/jjYd5SMhHbFJdBui9
} 


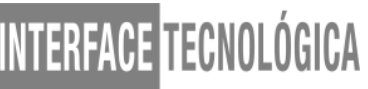

decomposto. As questões validam se os requisitos atendiam necessidades reais de Assistência de Enfermagem em Sala de Recuperação Pós-Anestésica, se são detalhados, se possuem conflitos, se são ambíguos uns com os outros e se são de fácil entendimento. Ao final, foi mostrado um fluxograma, com a finalidade dos especialistas verificarem os passos dos procedimentos.

O formulário para validação do protótipo (ii) cobriu 41 questões, 25 questões de múltipla escolha e 16 questões abertas, dívidas em 2 seções. A primeira Seção orienta a instalação do protótipo e a execução de algumas tarefas. As onze questões foram baseadas na proposta de Pressman e Maxim (2016), que verifica se há informações irrelevantes, se apresentam forma natural e lógica, ambiguidades e detalhamento adequado.

\subsection{Procedimento}

A avaliação do protótipo foi realizada utilizando como hardware smartphones de 7 polegadas e com sistema operacional Android versão 9. A primeira etapa consistiu em responder o formulário de revisão de requisitos pelos avaliadores. Primeiro, foi solicitado que o avaliador lesse sobre as orientações gerais. Em seguida, em cada seção do formulário, foi solicitado que os avaliadores abrissem um arquivo em anexo para visualizar os requisitos decompostos e posteriormente responder dez questões, sendo multipla escolha e aberta. No final do formulário, foi solicitado aos avaliadores a verificarem o fluxo das informações e a integração dos procedimentos decompostos.

$\mathrm{Na}$ segunda etapa, os avaliadores foram convidados executarem um conjunto de tarefas no protótipo. As atividades abrangeram inserir respostas de texto, múltipla escolha, seguir para a próxima tela ou voltar para a anterior, selecionar pacientes e escolher qual procedimento apresentar. Posteriormente, foi solicitado que os avaliadores respondessem ao formulário de avaliação do protótipo. $\mathrm{Na}$ abertura do questionário, foi apresentada as orientações gerais. Na sequência, foi apresentada 11 questões acerca de identificar se o protótipo apresenta uma ordem natural e lógica, se apresenta alguma informação irrelevante ou duplicada, pouco detalhada e se o fluxo está errado.

\subsection{Análise}

Neste trabalho foi usada como a métrica para avaliação o cálculo de concordância Kappa de Fleiss. Esse cálculo é uma extensão do Kappa de Cohen e é utilizado para verificar a concordância de mais de dois avaliadores quando realizam uma avaliação nominal ou 
ordinal em uma mesma amostra. A interpretação do coeficiente Kappa pode ser classificada como: i) concordância quase perfeita (valores entre 0,81 e 1,00); ii) concordância substancial, $(0,61$ e 0,80$)$; iii) concordância moderada $(0,41$ e 0,60$)$; iv) concordância fraca ou pequena $(0,21$ e 0,40$)$; v) concordância leve $(0,0$ e 0,20$)$, e; vi) nenhuma correlação quando forem menores do que zero (0), indicando ausência de acordo.

Para efetuar a análise, foi utilizado um software estatístico. A Tabela 3 apresenta o índice de concordância entre os avaliadores de cada procedimento decomposto na Revisão de requisitos $^{6}$. Observa-se que o item "Exames físicos" apresentou um índice de concordância moderada $(k=0,47)$. Um dos avaliadores apontou que alguns itens possuem conflito e que não estão adequadas as necessidades reais dos discentes, pois apresentam informações confusas. Os itens "Recebimento de informações" e "Índice de Aldrete e Kroulil", um dos avaliadores apontou informações adicionais a serem inseridas, apresentando um índice de concordância substâncial.

Tabela 3 - Índice de concordância de cada categoria para Revisão de requisitos

\begin{tabular}{|l|c|c|}
\cline { 2 - 3 } \multicolumn{1}{c|}{} & K-Fleiss & $\begin{array}{c}\text { Porcentagem de } \\
\text { concordância (\%) }\end{array}$ \\
\hline ABC & 1,00 & $100 \%$ \\
\hline Recebimento de informações & 0,75 & $87,50 \%$ \\
\hline Sinais Vitais & 1,00 & $100 \%$ \\
\hline Exames físicos & 0,47 & $75 \%$ \\
\hline Índice de Aldretee Kroulik & 0,75 & $87,50 \%$ \\
\hline Diagnósticos, Intervençõese Resultados de Enfermagem & 1,00 & $100 \%$ \\
\hline Prognóstico/Alta da SRPA & 1,00 & $100 \%$ \\
\hline Fluxo geral & $-0,33$ & $50 \%$ \\
\hline
\end{tabular}

Fonte: Próprio autor

No geral, os avaliadores/juízes consideraram os requisitos elicitados adequados, sendo observado um valor bruto consensual de $91.38 \%$. A concordância, verificada com auxílio do Kappa de Fleiss foi de $k=0,83,95 \%$ CI $[0,68,0,98]$, o que indica uma concordância quase perfeita, e assim não é possível rejeitar a $\mathbf{H 1}^{7}$.

Outra técnica utilizada para avaliação dos requisitos elicitados foi a utilização de um protótipo $^{8}$ pelos avaliadores. Ao utilizar o protótipo, ouve uma concordância bruta consensual de $90 \%$, em que os avaliadores acreditam que os requisitos foram elicitados de forma adequada. A concordância verificada com auxílio do Kappa Fleiss foi de $k=0,73,95 \%$ CI

\footnotetext{
${ }^{6}$ Os dados estão disponíveis em: https://bit.ly/3gersqk

${ }^{7} \mathrm{H} 1$ : Os avaliadores/juízes apresentaram um alto nível de concordância na Revisão de requisitos dos requisitos elicitados por meio do PC na análise de documentos

${ }^{8}$ Os dados estão disponíveis em: https://bit.ly/3qCrVb1
} 


\section{WTEERFAEETECNOLOGGCA}

$[0,11,1,00]$, o que indica uma concordância substancial, também não é possível rejeitar a $\mathrm{H}^{9}$.

Acredita-se que a diferença entre concordância da elicitação de requisitos de forma correta julgada pelos avaliadores seja devido a melhor visualização da informação. O protótipo apresenta as informações de maneira que os avaliadores conseguem executar os procedimentos em ambientes reais e "concretizado", possibilitando ver melhor o fluxo e os tipos de campos do formulário.

\section{AMEAÇAS À VALIDADE}

A fim de garantir que a maior parte dos requisitos foram elicitados de maneira adequada, foram selecionados avaliadores especialistas com formação em Enfermagem, com conhecimentos em Assistência de Enfermagem em Sala de Recuperação Pós-Anestésica. Porém, não pode ser descartada a ameaça em relação a opiniões e experiências pessoas dos avaliadores, que podem avaliar problemas de maneiras diferentes dos demais e assim afetar os resultados.

\section{DISCUSSÃO E CONCLUSÃO}

Para alguns sistemas, a análise de documentos como fonte para elicitação de requisitos é fundamental. Por exemplo, requisitos para o desenvolvimento de aplicativos para apoiar os procedimentos perioperatório pós-cirúrgico precisam ser baseados em protocolos prédefinidos por especialistas de enfermagem para um atendimento e avaliação adequada ao paciente. O nível de detalhe, fluxo das informações e sequência dos passos devem obedecer a sequência céfalo-podal. Assim, este estudo investiga o uso do Pensamento Computacional para auxiliar na análise de documentos de enfermagem. Foi proposto uma adaptação das etapas para análise de documentos proposto por O'Leary (2017), em que foi inserido os conceitos de decomposição, reconhecimento de padrões, abstração e desenho algorítimico, com uma pergunta norteadora sobre o que se procura e palavras-chaves previamente definidas.

\footnotetext{
${ }^{9} \mathrm{H} 2$ : Os requisitos elicitados apresentados no Protótipo se apresentaram adequados pelos avaliadores/juízes na análise de documentos
} 
Posteriormente, com os requisitos elicitados foi possível o desenvolvimento de um protótipo em Android para o apoio dos procedimentos perioperatórios pós-cirúrgico. Quatro avaliadores especialistas em Enfermagem Perioperatória Pós-Cirúrgica e docentes de uma Universidade Estadual Paranaense avaliaram os requisitos por meio da Revisão de Requisitos e de um Protótipo. Por meio da análise Kappa de Fleiss, os resultados mostraram um alto índice de concordância entre os avaliadores no documento de Revisão de Requisitos, e um índice de concordância substancial na concordância dos requisitos levantados e visualizados no Protótipo. Acredita-se que isso ocorreu devido a facilidade da análise dos requisitos por meio de um material mais "concreto", em que foi possível visualizar na prática.

No geral, os resultados desse estudo sugerem que o uso do Pensamento Computacional pode auxiliar na elicitação de requisitos por meio da análise de documentos, proporcionando maior índice de integração e detalhes das informações. Além disso, acreditase que este estudo contribua para a comunidade científica como meio sistematizado para a elicitação de requisitos por meio da análise de documentos, que comumente é realizado de forma $a d-h o c$, e muitas vezes os requisitos não são validados depois de elicitados.

\section{REFERÊNCIAS}

BOWEN, Glenn A. et al. Document analysis as a qualitative research method. Qualitative research journal, v. 9, n. 2, p. 27, 2009.

DENNIS, Alan; WIXOM, Barabara Haley; ROTH, Roberta. Systems Analysis and Design. John Wiley \& Sons, 2018.

O'LEARY, Zina. The essential guide to doing your research project. Sage, 2017.

PRESSMAN, Roger; MAXIM, Bruce. Engenharia de Software- $8^{\text {a }}$ Edição. McGraw Hill Brasil, 2016.

SELBY, Cynthia; WOOLLARD, John. Computational thinking: the developing definition. 2013.

SOBECC-Práticas Recomendadas SOBECC. 2017. Sociedade Brasileira de Enfermeiros de Centro Cirúrgico. Recuperação Anestésica e Centro de Material e Esterilização.

SOMMERVILlE, Ian. Engenharia de Software, 8 edição. Pearson, Addison Wesley, v. 8, n. 9, p. 10, 2011.

SOUZA, Talita Monteiro; CARVALHO, Rachel; PALADINO, Camila Moreira. Diagnósticos, prognósticos e intervenções de enfermagem na sala de recuperação pósanestésica. Revista SOBECC, v. 17, n. 4, p. 33-47, 2012. 
TSUMAKI, Toshihiko; TAMAI, Tetsuo. Framework for matching requirements elicitation techniques to project characteristics. Software Process: Improvement and Practice, v. 11, n. 5, p. 505-519, 2006.

ZIMMERMANN, Jussara et al. Proposta de aplicaçao e avaliaçao de conceitos do Pensamento Computacional em crianças hospitalizadas. In: Anais dos Workshops do Congresso Brasileiro de Informática na Educação. 2016. p. 1249.

ZOWGHI, Didar; COULIN, Chad. Requirements elicitation: A survey of techniques, approaches, and tools. In: Engineering and managing software requirements. Springer, Berlin, Heidelberg, 2005. p. 19-46. 